DEMONSTRATIO MATHEMATICA

Vol. XXXIII No $2 \quad 2000$

Gerd Herzog, Roland Lemmert

\title{
NONLINEAR FUNDAMENTAL SYSTEMS FOR LINEAR DIFFERENTIAL EQUATIONS IN FRÉCHET SPACES
}

\begin{abstract}
Let $E$ be a Fréchet space. We prove that $e x(E)=e x^{\prime}(E)$, that is that the IVP $u^{\prime}=A u+f, u(0)=u_{0}$ is always solvable if the homogeneous problem $u^{\prime}=A u$, $u(0)=u_{0}$ is always solvable (even if this solution is not unique). Moreover we prove that there is a continuous, in general nonlinear selection of solutions, which can be applied to prove an existence theorem for $u^{\prime}=A u+g(\cdot, u), u(0)=u_{0}$.
\end{abstract}

\section{Introduction}

Let $E$ be a real Fréchet space and $\left(p_{n}\right)_{n=1}^{\infty}$ a separating family of seminorms corresponding to the topology of $E$. Fix $T>0$, and let $C([0, T], E)$ be polynormed by $\left(q_{n}\right)$ with $q_{n}(u)=\max \left\{p_{n}(u(t)): t \in[0, T]\right\}$, let $E^{*}$ denote the topological dual space of $E$ endowed with the weak* topology $\sigma\left(E^{*}, E\right)$, and let $L(E)$ and $L\left(E^{*}\right)$ be the spaces of all continuous endomorphisms of $E$ and $E^{*}$, respectively. Moreover $A^{*} \in L\left(E^{*}\right)$ denotes the adjoint of $A \in L(E)$ with respect to the duality $\left\langle E, E^{*}\right\rangle$.

For $A \in L(E), f \in C([0, T], E)$ and $u_{0} \in E$ we consider the initial value problems (IVPs for short)

$$
\begin{aligned}
& u^{\prime}(t)=A u(t), \quad u(0)=u_{0} ; \\
& u^{\prime}(t)=A u(t)+f(t), \quad u(0)=u_{0} .
\end{aligned}
$$

It is well known that (1) can have no or more than one solution. Following the notation in [1] and [6] we define:

$\operatorname{ex}(E):=\left\{A \in L(E):(1)\right.$ is solvable on $\left.[0, T]\left(u_{0} \in E\right)\right\}$,

$\operatorname{ex}^{\prime}(E):=\left\{A \in L(E):(2)\right.$ is solvable on $\left.[0, T]\left(u_{0} \in E, f \in C([0, T], E)\right)\right\}$, unex $(E):=\left\{A \in L(E):(1)\right.$ is uniquely solvable on $\left.[0, T]\left(u_{0} \in E\right)\right\}$, $\ln (E):=\left\{A \in L(E): \exp (t A) u_{0}\right.$ is convergent $\left.\left(t \in \mathbb{R}, u_{0} \in E\right)\right\}$. 
The sets ex $\left(E^{*}\right)$, unex $\left(E^{*}\right)$ are defined analogously. Obviously $\ln (E) \subseteq$ unex $(E) \subseteq \operatorname{ex}(E) \subseteq L(E)$, and strict inclusion can occur in all possible situations, see [1], [6]. In case of $E=\omega$, the space of all real sequences, endowed with the topology of coordinatewise convergence, we have $\operatorname{unex}(\omega) \neq \operatorname{ex}(\omega)=L(\omega)$ and $\operatorname{ex}(\omega)=\operatorname{ex}^{\prime}(\omega)$ (see [3], [5] and [7]). In this paper we prove:

Theorem 1. Let $A \in \operatorname{ex}(E)$. Then $A \in \operatorname{ex}^{\prime}(E)$ and there is a continuous function $S: E \times C([0, T], E) \rightarrow C([0, T], E)$, such that $S\left(u_{0}, f\right)$ is a solution of (2).

Theorem 2. Let $A \in \operatorname{ex}(E)$ and $g:[0, T] \times E \rightarrow E$ continuous and compact. Then the IVP

$$
u^{\prime}(t)=A u(t)+g(t, u(t)), \quad u(0)=u_{0}
$$

is solvable on $[0, T]\left(u_{0} \in E\right)$.

REMARKS: 1). In Theorem 1 it is possible to get a selection $\widetilde{S}$ of solutions such that $\widetilde{S}(0,0)=0$. Just set $\widetilde{S}\left(u_{0}, f\right)=S\left(u_{0}, f\right)-S(0,0)$.

2). Since $\omega$ is a Montel space and since $L(\omega)$ can be represented as the space of all row-finite matrices (see for example [3]), Theorem 2 implies the solvability of IVPs for row-finite systems of differential equations $u^{\prime}=$ $A u+g(\cdot, u), u(0)=u_{0}$ with $g$ row-finite continuous and bounded. IVPs for nonautonomous row-finite systems $u^{\prime}=A(t) u$ can be locally unsolvable [4].

The main tool used to obtain the above results is that a continuous linear and surjective operator between Fréchet spaces has a continuous (in general nonlinear) right inverse (see for example Bourbaki, TVS II, Ch. 4, Proposition 12, [2]).

In the sequel, for $A \in \operatorname{ex}(E)$ let

$F:=\left\{u \in C([0, T], E): u\right.$ is differentiable and solves $\left.u^{\prime}(t)=A u(t)\right\}$.

The space $F$ is a closed subspace of $C([0, T], E)$, hence $F$ is a Fréchet space. The operator $P: F \rightarrow E$ defined by $P(u)=u(0)$ is linear and continuous, and $P$ is surjective since $A \in \operatorname{ex}(E)$. Now $P$ has a continuous right inverse, that is there is a continuous mapping $Q: E \rightarrow F$ with $P \circ Q=i d_{E}$.

It is well known that a linear continuous and surjective operator between Fréchet spaces has a linear right inverse if and only if its kernel has a topological complement (see for example [8], p. 109). In our case $P$ has a continuous linear right inverse if $A \in \operatorname{unex}(E)$. If $A \in \operatorname{ex}(E)$ but $A \notin \operatorname{unex}(E)$ in general there is no continuous linear right inverse, but there are examples for that a continuous linear right inverse can exist even in this case (see [1], Example 4.15 and $[6])$. 


\section{Proofs}

Proof of Theorem 1: Fix $f \in C([0, T], E)$ and let $H:[0, T] \times[0, T] \rightarrow E$ be defined by $H(t, s)=Q(f(t))(s)$. Observe that:

a) $H$ is continuous.

Indeed. To see this let us fix $(\tilde{t}, \tilde{s}) \in[0, T] \times[0, T]$ and a seminorm $q_{n}$. Since $Q$ and $f$ are continuous there exists $\delta>0$ such that

$$
t \in[0, T],|t-\tilde{t}|<\delta \Longrightarrow q_{n}(Q(f(t))-Q(f(\tilde{t})))<\varepsilon,
$$

hence

$$
p_{n}(Q(f(t))(s)-Q(f(\widetilde{t}))(s))<\varepsilon \quad(s \in[0, T]) .
$$

Moreover, the function $Q(f(\tilde{t}))(\cdot):[0, T] \rightarrow E$ is continuous. Therefore we can choose $\delta>0$ such that in addition

$$
p_{n}(Q(f(\widetilde{t}))(s)-Q(f(\widetilde{t}))(\widetilde{s}))<\varepsilon \quad(s \in[0, T],|s-\widetilde{s}|<\delta) .
$$

Thus we have

$$
p_{n}(H(t, s)-H(\tilde{t}, \tilde{s}))<2 \varepsilon \quad(t, s \in[0, T],|t-\tilde{t}|<\delta,|s-\tilde{s}|<\delta) .
$$

b) The derivative $H_{s}(t, s)$ exists for each $(t, s) \in[0, T] \times[0, T]$, and

$$
H_{s}(t, s)=A H(t, s) \quad(t, s \in[0, T]) .
$$

Hence $H_{s}:[0, T] \times[0, T] \rightarrow E$ is continuous.

c) The function $v:[0, T] \rightarrow E$ defined by

$$
v(t):=\int_{0}^{t} H(\tau, t-\tau) d \tau
$$

is differentiable on $[0, T]$ and $v^{\prime}(t)=A v(t)+f(t)(t \in[0, T])$.

Indeed. The integral in the definition of $v$ exists (as a Riemann integral) since $\tau \mapsto H(\tau, t-\tau)$ is continuous on $[0, t]$, and $v \in C([0, T], E)$ since $H$ is continuous on $[0, T] \times[0, T]$.

Let $\varphi \in E^{*}$. We have $\varphi \circ H \in C([0, T] \times[0, T], \mathbb{R})$ and $(\varphi \circ H)(t, s)$ is continuously differentiable in $s$. Hence

$$
\begin{aligned}
\frac{d}{d t}\left(\int_{0}^{t} \varphi(H(\tau, t-\tau)) d \tau\right) & =\varphi(H(t, 0))+\int_{0}^{t} \frac{d}{d t} \varphi(H(\tau, t-\tau)) d \tau \\
& =\varphi(Q(f(t))(0))+\varphi\left(A \int_{0}^{t} H(\tau, t-\tau) d \tau\right) \\
& =\varphi(f(t)+A v(t)) \quad(t \in[0, T]) .
\end{aligned}
$$


This proves that $v$ is weakly differentiable with weak derivative $v^{\prime}=A v+f$. Hence $v^{\prime} \in C([0, T], E)$ and therefore $v$ is differentiable on $[0, T]$. Thus $v$ solves (2) with $u_{0}=0$;

d) The function $u:[0, T] \rightarrow E$, defined by

$$
u(t):=Q\left(u_{0}\right)(t)+\int_{0}^{t} Q(f(\tau))(t-\tau) d \tau
$$

is a solution of (2).

e) Let $S\left(u_{0}, f\right)$ be the solution of (2) selected in d). This function $S$ : $E \times C([0, T], E) \rightarrow C([0, T], E)$ is continuous.

To prove this let $d: C([0, T], E) \times C([0, T], E) \rightarrow \mathbb{R}$ denote the usual metric corresponding to $\left(q_{n}\right)$. First note that $S(\cdot, f): E \rightarrow C([0, T], E)$ is continuous since $Q$ is continuous. Next, let $\left(f_{k}\right)$ be a convergent sequence in $C([0, T], E)$ with limit $f \in C([0, T], E)$. Then

$$
Q\left(f_{k}(t)\right) \rightarrow Q(f(t))(k \rightarrow \infty) \text { in } C([0, T], E) \text { uniformly on }[0, T]:
$$

Otherwise there would be an $\varepsilon_{0}>0$ and a subsequence $\left(f_{k_{j}}\right)$ of $\left(f_{k}\right)$ and corresponding $t_{j} \in[0, T]$ such that

$$
d\left(Q\left(f_{k_{j}}\left(t_{j}\right)\right), Q\left(f\left(t_{j}\right)\right)\right) \geq \varepsilon_{0} .
$$

Without loss of generality let $t_{j} \rightarrow t_{0}(j \rightarrow \infty)$.

Since $\lim _{j \rightarrow \infty} f_{k_{j}}\left(t_{j}\right)=f\left(t_{0}\right)=\lim _{j \rightarrow \infty} f\left(t_{j}\right)$ we have

$$
\lim _{j \rightarrow \infty} Q\left(f_{k_{j}}\left(t_{j}\right)\right)=Q\left(f\left(t_{0}\right)\right) \text { in } C([0, T], E)
$$

which is a contradiction to $(*)$. This means that for $H_{k}, H:[0, T] \times[0, T] \rightarrow$ $E$, defined by $H_{k}(t, s)=Q\left(f_{k}(t)\right)(s), H(t, s)=Q(f(t))(s)$,

$$
H_{k} \rightarrow H(k \rightarrow \infty) \text { uniformly on }[0, T] \times[0, T] \text {. }
$$

Hence

$$
\int_{0}^{t} H_{k}(\tau, t-\tau) d \tau \rightarrow \int_{0}^{t} H(\tau, t-\tau) d \tau \quad(k \rightarrow \infty)
$$

uniformly on $[0, T]$.

Proof of Theorem 2. Let $S: E \times C([0, T], E) \rightarrow C([0, T], E)$ be as in Theorem 1 and consider the operator $K: C([0, T], E) \rightarrow C([0, T], E)$ defined by

$$
K(v)(t):=S\left(u_{0}, g(\cdot, v)\right)(t)=Q\left(u_{0}\right)(t)+\int_{0}^{t} Q(g(\tau, v(\tau)))(t-\tau) d \tau .
$$

$K$ is continuous, since $v_{k} \rightarrow v$ implies $g\left(\cdot, v_{k}\right) \rightarrow g(\cdot, v)(k \rightarrow \infty)$ in $C([0, T], E)$. According to the assumptions, there is a compact $M \subseteq E$ 
such that

$$
g([0, T] \times E) \subseteq M
$$

Fix $t \in[0, T]$. For each $v \in C([0, T], E)$ and $\tau \in[0, t]$ we have

$$
Q(g(\tau, v(\tau))) \in Q(M),
$$

(where $Q(M) \subseteq C([0, T], E)$ is compact), and therefore there is a compact $M_{1} \subseteq E$ such that

$$
Q(g(\tau, v(\tau)))(t-\tau) \in M_{1} \quad(0 \leq \tau \leq t \leq T) .
$$

The mean value theorem gives

$$
\begin{aligned}
K(v)(t) & \in Q\left(u_{0}\right)(t)+t \cdot \overline{\operatorname{conv}}\left(M_{1} \cup\{0\}\right) \\
& \subseteq Q\left(u_{0}\right)([0, T])+T \cdot \overline{\operatorname{conv}}\left(M_{1} \cup\{0\}\right) \\
& =: M_{2},
\end{aligned}
$$

where $M_{2} \subseteq E$ is compact, so

$$
\overline{\{K(v)(t): v \in C([0, T], E)\}}
$$

is a compact subset of $E$ for each $t \in[0, T]$. Moreover

$$
\{K(v): v \in C([0, T], E)\}
$$

is equicontinuous since the derivatives $(K(v))^{\prime}(t)$ are contained in the bounded (even compact) set $A M_{2}+M$. Hence

$$
\overline{\{K(v): v \in C([0, T], E)\}}
$$

is a compact subset of $C([0, T], E)$ according to Arzelà-Ascoli's theorem. Tychonov's fixed point theorem now implies that there is a function $u \in$ $C([0, T], E)$ such that $K(u)=u$, and $u$ solves $(3)$.

\section{Example}

Beside row-finite systems, as outlined in the introduction, our results can be applied for example to the following equation:

Consider the Montel-Fréchet space $E=C^{\infty}([0,1], \mathbb{R})$ (for example $p_{n}(x)$ $=\max \left\{\left|x^{(n)}(s)\right|: s \in[0,1]\right\}$ for $n \geq 0$ ), and let $A \in L(E)$ defined by $A x=x^{\prime}$. It is known [6] that $A \in \operatorname{ex}(E)$ but $A \notin u \operatorname{unex}(E)$.

For $h:[0,1] \times \mathbb{R} \rightarrow \mathbb{R}$ continuous and bounded let $g:[0,1] \times E \rightarrow E$ be the continuous and compact function defined by

$$
g(t, x)(s)=\int_{0}^{t} h\left(t, x^{\prime \prime}(\sigma)\right) d \sigma .
$$

Note that for each $(t, x)$ the function $g(t, x)$ is constant. 
According to Theorem 2 each IVP $u^{\prime}=A u+g(\cdot, u), u(0)=u_{0}$ is solvable on $[0,1]$, which implies that the problem

$$
v_{t}(t, s)=v_{s}(t, s)+\int_{0}^{t} h\left(t, v_{s s}(t, \sigma)\right) d \sigma, \quad v(0, s)=v_{0}(s)
$$

with $v_{0} \in C^{\infty}([0,1], \mathbb{R})$ has a solution $v:[0,1] \times[0,1] \rightarrow \mathbb{R}$ such that $v(t, s)$ is $C^{1}$ in $t, C^{\infty}$ in $s$ and all partial derivatives are continuous on $[0,1] \times[0,1]$.

\section{References}

[1] V. I. Bogachev, Deterministic and stochastic differential equations in infinite dimensional spaces, Acta Appl. Math. 40 (1995), 25-93.

[2] N. Bourbaki, Topological Vector Spaces, Chapters 1-5, Springer-Verlag, BerlinHeidelberg-New York-London-Paris-Tokyo, 1987.

[3] G. Herzog, On ordinary linear differential equations in $\mathbb{C}^{J}$, Demonstratio Math. 28 (1995), 383-398.

[4] G. Herzog, On linear time-dependent row-finite systems of differential equations, Travaux Math. Sem. Math. Luxemb. 8 (1996), 167-176.

[5] R. Lemmert, Ä. Weckbach, Charakterisierungen zeilenendlicher Matrizen mit abzählbarem Spektrum, Math. Z. 188 (1984), 119-124.

[6] S. G. Lobanov, On the solvability of linear ordinary differential equations in locally convex spaces, Moscow Univ. Math. Bull. 25 (1980), 1-5.

[7] S. A. Shkarin, Some results on solvability of ondinary linear differential equations in locally convex spaces, Math. USSR Sbornik 71 (1992), 29-40.

[8] F. Trèves, Locally Convex Spaces and Linear Partial Differential Equations, Springer-Verlag, Berlin-Heidelberg-New York, 1967.

MATHEMATISCHES INSTITUT I

UNIVERSITĀT KARLSRUHE

D-76128 KARLSRUHE, GERMANY

E-mail: Gerd.HerzogOmath.uni-karlsruhe.de

E-mail: Roland.Lemmert Omath.uni-karlsruhe.de

Received July 13, 1999. 